\title{
Gas Sensors Based on Single-Walled Carbon Nanotube Field-Effect Transistor
}

\author{
Liangming Wei ${ }^{1, *}$, Haiyan Chen ${ }^{1}$, Jian Wang ${ }^{1}$, WeienYuang, ${ }^{2, * *}$, \\ Jiang $\mathrm{Zhao}^{1}$, Dong $\mathrm{Xu}^{1}$ and Yafei Zhang ${ }^{1, * * *}$ \\ ${ }^{1}$ Key Laboratory for Thin Film and Microfabrication of the Ministry of Education, \\ Institute of Micro/Nano Science and Technology, Shanghai Jiao Tong University, \\ Shanghai 200240, P. R. China \\ ${ }^{2}$ School of Pharmacy, Shanghai Jiao Tong University, Shanghai 200240, P. R. China
}

(Received June 1, 2013; accepted November 7, 2013)

Key words: carbon nanotubes, field-effect transistors, gas sensor, self-assembly, dimethyl methylphosphonate

Single-walled carbon nanotube (SWCNT) gas sensors with field-effect-transistor (FET) structure have received much attention owing to their excellent properties such as high sensitivity, small size, room-temperature operation, and self-desorption by gate bias. In this study, SWCNT-FET was constructed via the self-assembly of a uniform SWCNT film on a $\mathrm{Si} / \mathrm{SiO}_{2}$ substrate, which exhibited a large current on/off ratio of up to $10^{5}$. This SWCNT-based FET could be used as gas sensors. Upon exposure to dimethyl methylphosphonate (DMMP, simulant of nerve agent sarin), the current of the device decreased and the threshold voltage shifted toward the negative gate voltage direction. This sensor could easily detect DMMP down to $1 \mathrm{ppm}$ concentration. This sensor could also be $100 \%$ recovered when a gate voltage of $15 \mathrm{~V}$ is applied to the device.

\section{Introduction}

Single-walled carbon nanotubes (SWCNTs) are nearly ideal one-dimensional (1D) quantum wires with every atom on the surface, and they possess high aspect ratio, good environmental stability, and excellent mechanical and electronic properties. These features make SWCNTs ideal sensing materials for compact, low-cost, lowpower, and portable gas-sensing devices. ${ }^{(1-6)}$ Upon exposure to some electron-donating or electron-withdrawing molecules (such as $\mathrm{NH}_{3}$ and $\mathrm{NO}_{2}$ ), the electrical conductance of SWCNTs is changed owing to charge transfer between SWCNT and the absorbed molecules. ${ }^{(4-10)}$ Multiple types of SWNT-based gas-sensing device, such as chemiresistors, chemicapacitors, and field-effect transistors (FET), have been developed for sensing

${ }^{*}$ Corresponding author: e-mail: lmwei@sjtu.edu.cn

${ }^{* *}$ Corresponding author: e-mail: pharmacy_ywe@126.com

${ }^{* * *}$ Corresponding author: e-mail: yfzhang@sjtu.edu.cn 
application, but FET is very attractive because it can provide an additional gate electrode to amplify the detection signal. ${ }^{(4,10,11)}$ Kong et al. first demonstrated that the electrical conductance of SWCNTs in the SWCNT-FET could change significantly upon exposure to $\mathrm{NO}_{2}$ and $\mathrm{NH}_{3}$ gases. ${ }^{(4)}$ Later, SWCNT gas sensors for detecting sub-ppm concentrations of $\mathrm{NO}_{2}, \mathrm{NH}_{3}, \mathrm{H}_{2}, \mathrm{CO}$, and $\mathrm{CH}_{4}$ were reported. ${ }^{(12-18)}$ Recently, several groups have made efforts towards developing SWCNT-based sensors for the detection of chemical weapon agents (CWAs). Lee et al. prepared a chemical SWCNT sensor in which SWCNTs were deposited by alternating-current dielectrophoresis, and found that this sensor showed high sensitivity to dimethyl methylphosphonate (DMMP) and thionyl chloride $\left.\left(\mathrm{SOCl}_{2}\right)\right)^{(19)}$ Cattanach et al. fabricated a flexible sensor by depositing an SWCNT network on polyethylene terephthalate (PET) substrates for the detection of DMMP and diisopropyl methylphosphonate (DIMP). ${ }^{(20)}$ Snow et al. demonstrated the use of SWCNTs for both chemiresistors and chemicapacitors for the sensing of various chemical vapors, such as DMMP and dinitrotoluene (DNT). ${ }^{(21,22)}$

So far, two main methods have been reported for depositing SWCNTs across electrodes for the fabrication of gas sensors. ${ }^{(23)}$ One is to directly grow CNTs on the sensor platform via chemical vapor deposition (CVD). This method involves high temperature, complex processes and low yields. The other is to drop-cast an SWCNT solution onto a prefabricated electrode surface. Solution-casting techniques, such as microsyringes and dielectrophoresis, are more reproducible, less expensive and have higher yields. However, these methods often suffer from poor electrical contact between nanotubes and electrodes. In this study, a facile and controllable method has been developed for the deposition of SWCNT networks. The advantage of this method is that it can be used for the fabrication of sensors at a wafer scale. The deposited SWCNTs can be absorbed firmly on the amino-terminated $\mathrm{Si} / \mathrm{SiO}_{2}$ wafer substrate, resulting in good electrical contact between SWCNTs and Au electrodes. Combined with conditional lithography and lift-off processes, SWCNT-FET gas sensors were made. The sensing behaviors of the gas sensor toward DMMP were investigated at room temperature.

\section{Materials and Methods}

\subsection{Materials}

All chemicals used in our experiments were of analytical reagent grade and were directly used without further purification. SWCNTs synthesized by the arc discharge method were used in this work. SWCNTs were purified by air oxidation at $365^{\circ} \mathrm{C}$ for $30 \mathrm{~min}$, followed by refluxing with a 3:1 mixture of concentrated sulfuric acid and nitric acid at $80{ }^{\circ} \mathrm{C}$ for $30 \mathrm{~min}$. During this process, the carboxyl groups were introduced on the surface of SWCNTs. The products were separated from the solution and washed three times with distilled water and ethanol, respectively. The final purified SWCNTs have a purity of about 95\%. DMMP and 3-aminopropyltrimethysilane (APS) were purchased from Sigma-Aldrich. Deionized water was used throughout all processes.

\subsection{SWCNT network deposition and sensor fabrication}

Our sensors were fabricated on n-doped silicon substrates (as a gate electrode) covered with $400 \mathrm{~nm}$ of thermally grown $\mathrm{SiO}_{2}$. This $\mathrm{Si} / \mathrm{SiO}_{2}$ wafer substrate was 
ultrasonically rinsed with toluene, acetone, ethanol, and deionized water for $10 \mathrm{~min}$ in turn, and then immersed in a Piranha solution $\left(\mathrm{H}_{2} \mathrm{SO}_{4}: \mathrm{H}_{2} \mathrm{O}_{2}=3: 1\right)$ for $2 \mathrm{~h}$ at $85^{\circ} \mathrm{C}$ to make its surface hydrophilic. The pretreated substrate was immersed in APS aqueous solution (APS:deionized water $=0.5 \mathrm{ml}: 50 \mathrm{ml}$ ) for $2 \mathrm{~h}$ and then kept in a vacuum evaporator at $120^{\circ} \mathrm{C}$ for $1 \mathrm{~h}$ to form the amino-terminated monolayer on the surface of the $\mathrm{Si} / \mathrm{SiO}_{2}$ substrate. The purified SWCNTs were ultrasonically dispersed in deionized water for $2 \mathrm{~h}$ and then centrifuged at $10000 \mathrm{rpm}$ for $20 \mathrm{~min}$, and the obtained stable upper suspension was taken for further deposition, as shown in Fig. 1. The pretreated silicon substrate modified with an APS monolayer was immersed in the SWCNT suspension, followed by rinsing with ethanol and drying with the aid of nitrogen flow. Zeta potential measurements for the SWCNT suspension were carried out using a zeta plus analyzer (Zetasizer, Malvern, UK). The morphology measurements of the deposited SWCNTs were performed by emission scanning electron microscopy (SEM, Zeiss Ultra 55 , Germany) at an acceleration voltage of $5 \mathrm{kV}$.

Using conventional microfabrication procedures, the SWCNT-based FET gas sensor was fabricated. The interdigitated electrode fingers were made by sputtering $25 \mathrm{~nm}$ $\mathrm{Ti}$ and $140 \mathrm{~nm} \mathrm{Au}$ onto a patterned photoresist mold. A lift-off process was further introduced to remove the photoresist. Finally, the electrodes were sonicated in ethanol,

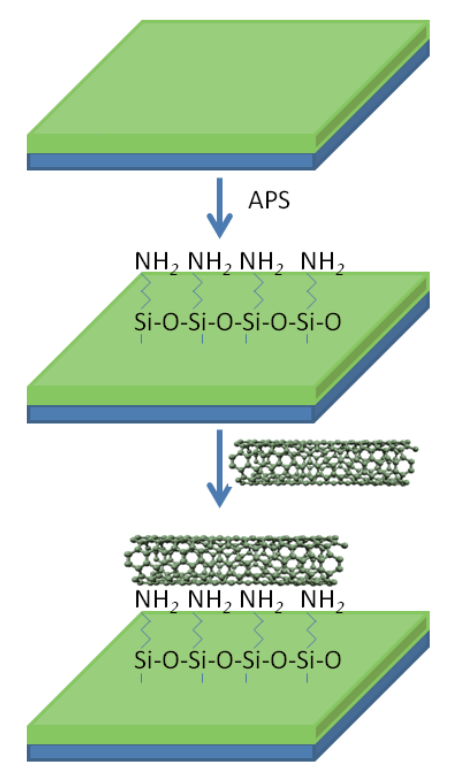

Fig. 1. (Color onlilne) Schematic diagram of APS-assisted SWCNT deposition on $\mathrm{Si} / \mathrm{SiO}_{2}$ substrate with amino-terminated monolayer on surface. 
washed with deionized water and then annealed at $200{ }^{\circ} \mathrm{C}$ for $1 \mathrm{~h}$ under vacuum. The devices were measured with an Agilent 4156C semiconductor parameter analyzer. To prepare semiconducting SWCNT-FET, the metallic SWCNTs (m-SWCNT) were removed from the SWCNT network using a current-induced electrical breakdown method as previously reported.(24,25) During this process, $V_{\text {sd }}$ was swept from 0 to $20 \mathrm{~V}$ while $V_{\mathrm{g}}$ was kept at $5 \mathrm{~V}$. A drop in the current at about $17 \mathrm{~V}$ was observed during the first $V_{\mathrm{ds}}$ scan owing to the breakdown of m-SWCNTs. The $V_{\mathrm{ds}}$ scan was repeated several times until a constant current was observed when $V_{\text {sd }}$ was swept from 0 to $20 \mathrm{~V}$.

\section{Results and Discussion}

For the fabrication of the SWCNT gas sensor, we first introduced carboxyl groups onto the surface of SWCNTs via an oxide process. The zeta potential of the SWCNT suspension in water was measured to be $-45.6 \mathrm{mV}$, which demonstrates that the SWCNTs are negatively charged in water. The SWCNTs could be absorbed and subsequently deposited on the amino-terminated silicon substrate via electrostatic interaction (Fig. 1). The interdigitated electrode arrays with a finger length of $110 \mu \mathrm{m}$ and a gap size of $5 \mu \mathrm{m}$ were patterned onto the deposited SWCNTs [Fig. 2(a)]. The SEM image shows that the SWCNTs are uniformly distributed on the silicon substrate and bridge the interdigitated electrodes [Fig. 2(b)].

Figure 3 shows the schematic of the SWCNT gas sensors with the FET structure and their optical images. The SWCNT-FET sensors provide an additional gate electrode to amplify the detection signal, but the coexistence of m-SWCNT in the SWCNT network (about 1/3) compromises the excellent performance of the SWCNT-FET because small shifts of the Fermi level in m-SWCNTs do not result in a substantial change in the density of state at the Fermi level, and thus, in the charge carriers in the nanotubes. ${ }^{(26,27)}$ Thus, the enriched semiconducting SWCNTs (s-SWCNTs) are preferred for the fabrication

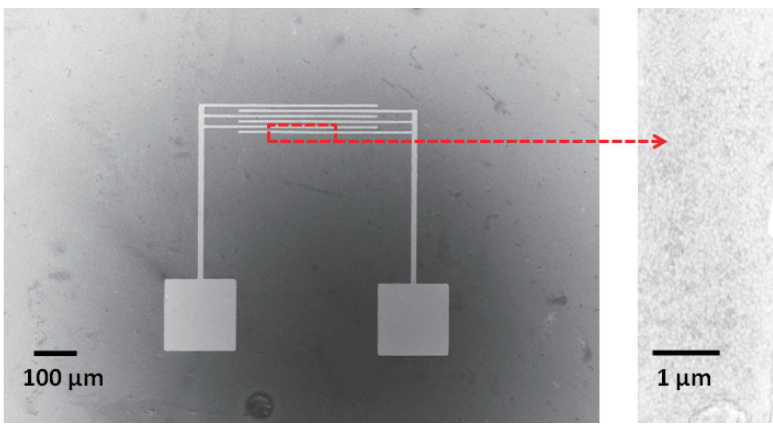

(a)

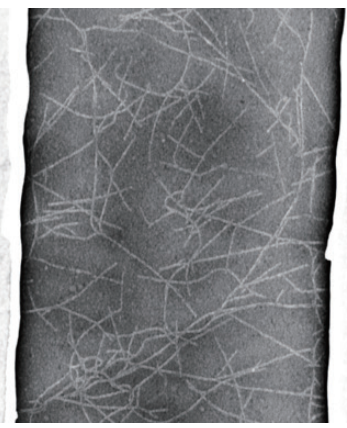

(b)

Fig. 2. (Color online) (a) $\mathrm{SEM}$ image of structure of Au electrode fabricated on $\mathrm{Si} / \mathrm{SiO}_{2}$ substrate. In this figure, the electrode is not connected to a power source. The white square shows $\mathrm{Au}$ electrodes used for the connected power source. (b) Enlarged SEM image of (a). The dotted rectangle indicates the place that is enlarged. In (b), the SWCNTs that bridge the Au electrodes can be seen. 
of SWCNT-based sensors. We prepared pure s-SWCNT-FET by selectively removing the m-SWCNTs from the SWCNT network using an electrical breakdown method as previously reported. $\left.{ }^{24,25}\right)$ Figure 4 shows the transfer characteristic of the SWCNT-FET with the electrode spacing of $5 \mu \mathrm{m}$. It could be found that the device current on/off ratio reached $10^{5}$, suggesting that the current was dominated by the s-SWCNTs when the electrode spacing was $5 \mu \mathrm{m}$.

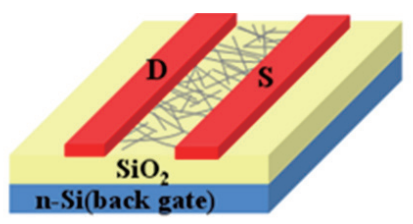

(a)

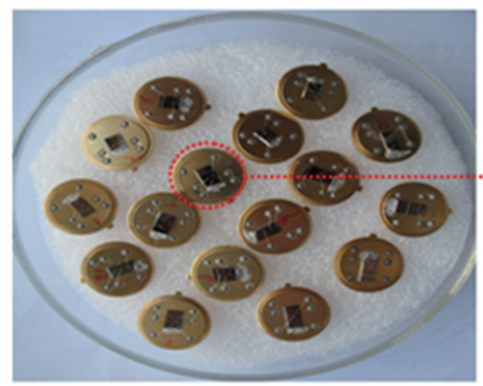

(c)

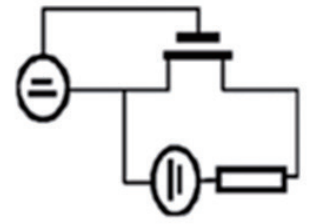

(b)

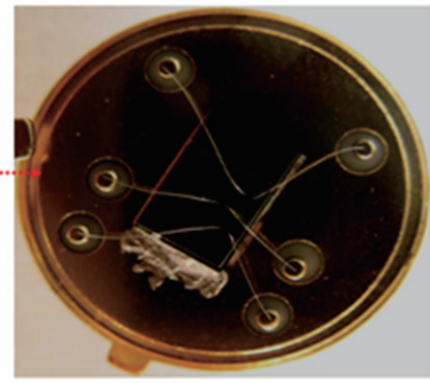

(d)

Fig. 3. (Color online) Schematic illustration of SWCNT-FET sensors and their optical images.

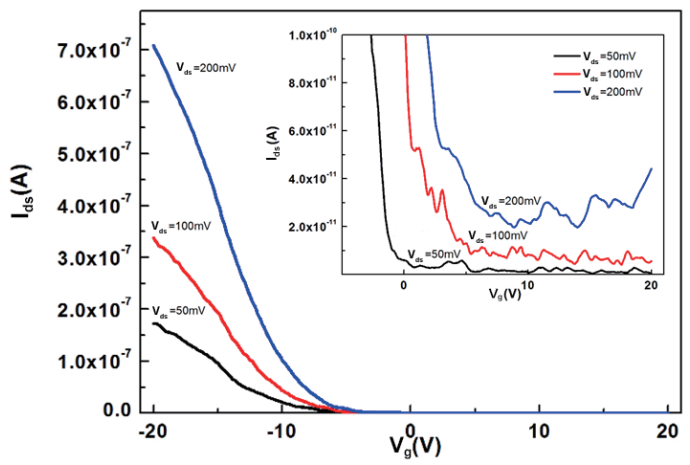

Fig. 4. (Color online) Transfer characteristic of SWCNT-FET. The plot on the right inset is the enlarged section where $V_{\mathrm{g}}$ is from -5 to $20 \mathrm{~V}$. The electrode spacing is $5 \mu \mathrm{m}$. 
Figure 5 shows the plot of the source-drain current $\left(I_{\mathrm{ds}}\right)$ versus gate voltage $\left(V_{\mathrm{g}}\right)$ measured under the background gas $\left(\mathrm{N}_{2}\right)$ and 1,10 , and 100 ppm DMMP. The sensors were put in a chamber specifically fabricated for the tests and were exposed to the gas for 5 min. Figure 5 shows that the device exhibits a p-type behavior as $V_{\mathrm{g}}$ is swept from -20 to $+20 \mathrm{~V}$. Upon exposure to DMMP gas, the transistor threshold voltage shifted to the negative voltage direction and the $I_{\mathrm{ds}}$ decreased in the negative voltage direction, while the $I_{\mathrm{ds}}$ increased in the positive voltage direction. The fact that the transfer curve shifted to a more negative gate voltage direction upon exposure to DMMP indicated that the adsorbed DMMP donated electrons to SWCNT.(4) These donated electrons need to be compensated from the gate voltage by the extra hole. The same curves can be observed when different concentrations of DMMP were introduced.

The sensor response $(\Delta R)$ in conductance is defined as

$$
R=\frac{\Delta R}{R}=\frac{R_{1}-R_{0}}{R_{0}}
$$

where $R_{0}$ and $R_{1}$ are the device resistances when the device was exposed to background gas and DMMP gas, respectively. Figure 6 shows the plot of the sensitivity versus time measured under different concentrations of DMMP. When $\Delta R$ reached the saturation value, a positive gate voltage pulse $\left(V_{\mathrm{g}}=15 \mathrm{~V}\right)$ was applied to refresh the gas sensor. Figure 6 shows that the SWCNT-FET sensor is very sensitive toward DMMP. When DMMP concentrations were 1,10 , and $100 \mathrm{ppm}$, the $\Delta R_{\mathrm{s}}$ were 20,38 , and $55 \%$, respectively, with a response time of about $300 \mathrm{~s}$. When adding a positive bias to the desorption of DMMP, the device can be recovered quickly. The sensing cycle was repeated several times and the devices performed very well. The reason for the desorption of DMMP molecules from the surface of SWCNT by a positive bias may be that the Coulomb interaction between the DMMP and the negative charge induced by the gate bias lowers the desorption barrier sufficiently to refresh the sensor at room

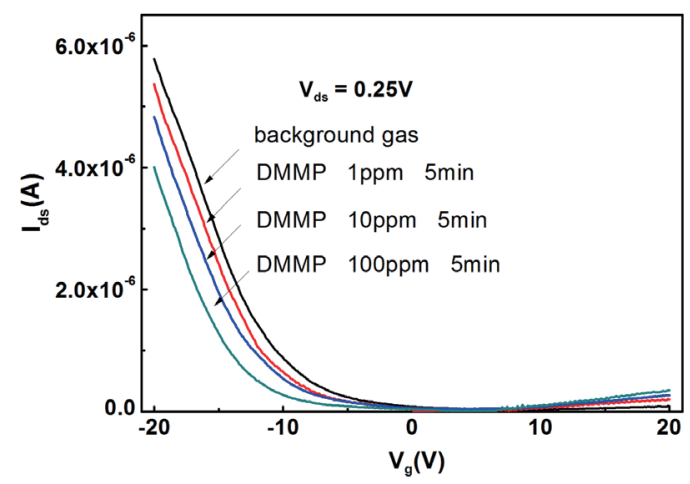

Fig. 5. (Color online) Transfer curve for SWCNT-FET sensor response to different DMMP concentrations (the source-drain voltage $V_{\mathrm{ds}}$ was $500 \mathrm{mV}$ ). The DMMP concentrations are 1, 10, and $100 \mathrm{ppm}$ and the exposure time is $5 \mathrm{~min}$. 
temperature. ${ }^{(28)}$

The sensitivity and selectivity of the sensors to some interfering organic vapors including water, methanol, benzene, acetone, dichloromethane, hexane and chloroform were also investigated. Figure 7 shows that the SWCNT-FET sensor exhibits excellent selectivity toward DMMP when exposed to those interfering vapors. For example, the conductance change of the sensors upon exposure to $1 \mathrm{ppm}$ DMMP was $20.5 \%$, whereas that change was only $2.1 \%$ upon exposure to $3020 \mathrm{ppm}$ ( $1 \%$ of the saturated vapor) acetone. This makes our sensor on ideal candidate for the ultratrace detection of DMMP.

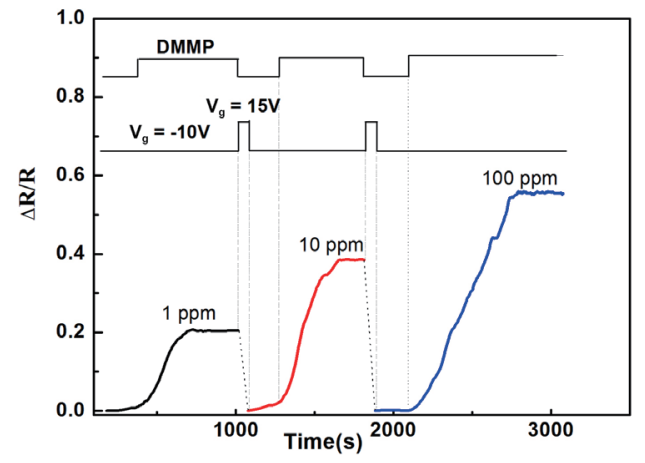

Fig. 6. (Color online) Normalized conductance change $\left(\Delta R / R_{0}\right)$ versus time when $V_{\mathrm{ds}}$ is $300 \mathrm{mV}$. When the conductance change reached the saturation value, a positive gate voltage pulse $\left(V_{\mathrm{g}}=15 \mathrm{~V}\right)$ with a duration of $60 \mathrm{~s}$ was applied to refresh the gas sensor.

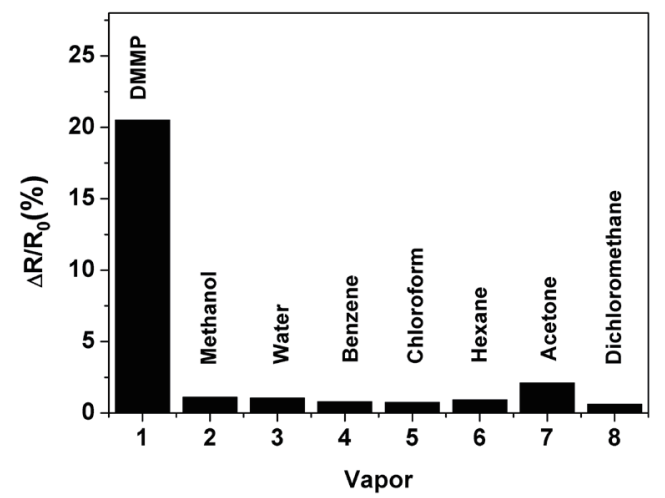

Fig. 7. Conductance change $\left(\Delta R / R_{0}\right)$ of SWNT-FET sensor upon exposure to DMMP and some interfering organic vapors. The interfering organic vapors were diluted to $1 \%$ of the saturated vapor and the concentration of the DMMP was $1 \mathrm{ppm}$. 


\section{Conclusions}

SWCNT-FET gas sensors were constructed via the self-assembly of a uniform SWCNT film on a $\mathrm{Si} / \mathrm{SiO}_{2}$ substrate, which exhibited a large current on/off ratio of $10^{5}$. When this sensor was exposed to DMMP vapor, the threshold voltage of the device moved toward the negative gate voltage direction and the conductance significantly decreased. Using this sensor, it is easy to detect DMMP at $1 \mathrm{ppm}$ concentration with the response time of less than $5 \mathrm{~min}$, without the need of preconcentrating the analytes. This sensor could also be $100 \%$ recovered when a gate voltage of $15 \mathrm{~V}$ is applied to the device, which is very important for practical application.

\section{Acknowledgements}

This work was financially supported by the National Natural Science Foundation of China (No. 51272155) and the Foundation for SMC Excellent Young Teachers in Shanghai Jiao Tong University.

\section{References}

1 H. X. Tang, M. Yan, H. Zhang, S. Z. Li, X. F. Ma, M. Wang and D. Yang: Sens. Actuators, B 114 (2006) 910.

2 F. Kreupl, A. P. Graham, G. S. Duesberg, W. Steinhögl, M. Liebau, E. Unger and W. Hönlein: Microelectron. Eng. 64 (2002) 399.

3 J. Suehiro, G. Zhou, H. Imakiire, W. Ding and M. Hara: Sens. Actuators, B 108 (2005) 398.

4 J. Kong, N. Franklin, C. Zhou, M. Chapline, S. Peng, K. Cho and H. Dai: Science 287 (2000) 622.

5 E. S. Snow, F. K. Perkins, E. J Houser, S. C. Badescu and T. L. Reinecke: Science 307 (2005) 1942.

6 A. Star, T. R. Han, V. Joshi, J. C. P. Gabriel and G. Grüner: Adv. Mater. 16 (2004) 2049.

7 L. M. Wei, D.W. Shi, P. Y. Ye, Z. Q. Dai, H.Y. Chen, C. X. Chen, J. Wang, L.Y. Zhang, D. Xu, Z. Wang and Y. F. Zhang: Nanotechnology 22 (2011) 425501.

8 P. Qi, O. Vermesh, M. Grecu, A. Javey, Q. Wang, H. Dai, S. Peng and K. Cho: Nano Lett. 3 (2003) 347.

9 L. Valentini, I. Armentano, J. M. Kenny, C. Cantalini, L. Lozzi and S. Santucci: Appl. Phys. Lett. 82 (2003) 961.

10 J. P. Novak, E. S. Snow, E. J. Houser, D. Park, J. L. Stepnowski and R. A. McGill: Appl. Phys. Lett. 83 (2003) 4026.

11 Y. Y. Wang, Z. H. Zhou, Z. Yang, X. H. Chen, D. Xu and Y. F. Zhang: Nanotechnology 20 (2009) 345502.

12 J. Zhao, A. Buldum, J. Han and J. P. Lu: Nanotechnology 13 (2002) 195.

13 L. Dai, P. Soundarrajan and T. Kim: Pure Appl. Chem. 74 (2002) 1753.

14 H. Chang, J. D. Lee, S. M. Lee and Y. H. Lee: Appl. Phys. Lett. 79 (2001) 3863.

15 W. S. Cho, S. I. Moon, Y. D. Lee, Y. H. Lee, J. H. Park and B. K. Ju: IEEE Electron Device Lett. 26 (2005) 498.

16 Q. Zhao, Z. Gan and Q. Zhuang: Electroanalysis 14 ( 2002) 1609.

17 A. Star, V. Joshi, S. Skarupo, D. Thomas and J. C. P. Gabriel: J. Phys. Chem. B. 110 (2006) 21014. 
18 D. R. Kauffman and A. Star: Angew. Chem. Int. Ed. 47 (2008) 6550.

19 C. Y. Lee, S. Baik, J. Zhang, R. I. Masel and M. S. Strano: J. Phys. Chem. B. 110 (2006) 11055 .

20 K. Cattanach, R. D. Kulkarni, M. Kozlov and S. K. Manohar: Nanotechnology 17 (2006) 4123.

21 E. S. Snow and F. K. Perkins: Nano Lett. 5 (2005) 2414.

22 E. S. Snow, F. K. Perkins, E. J. Houser, S. C. Badescu and T. L. Reinecke: Science 307 (2005) 1942.

23 T. Zhang, S. Mubeen, N. V. Myung and A. M. Deshusses: Nanotechnology 19 (2008) 332001.

24 P. G. Collins, M. S. Arnold and P. Avouris: Science 292 (2001) 706.

25 C. X. Chen, D. Xu, E. S. W. Kong and Y. F. Zhang: IEEE Electron Device Lett. 27 (2006) 852.

26 E. L. Gui L. J. Li, P. S.Lee, A. Lohani, S. G. Mhaisalkar, Q. Cao, S. J. Kang, J. A. Rogers, N. C. Tansil and Z. Gao: Appl. Phys. Lett. 89 (2006) 232104.

27 C. Y. Lee and M. S.Strano: Langmuir 21 (2005) 5192.

28 J. P. Novak, E. S. Snow, E. J. Houser, D. Park, J. L. Stepnowski and R. A. McGill: Appl. Phys. Lett. 83 (2003) 4026. 